\title{
La problématique de la gouvernance et du principe environnemental de responsabilité
}

\section{A problemática da governança e do princípio ambiental da responsabilidade}

The problem of governance and the environmental principle of responsibility

\section{Grace Ladeira Garbaccio ${ }^{1}$ \\ Michel Prieur ${ }^{2}$ \\ Marie Tanchon ${ }^{3}$ \\ Samantha Ribeiro Meyer- Pflug ${ }^{4}$}

Résumé : La gouvernance, symbole d'une nouvelle modernité dans les modes d'action publique et le gouvernement des entreprises, constitue le $4^{e}$ pilier du développement durable. Les liens entre la gouvernance et le principe environnemental de la responsabilité apparaissent inextricables. De ce fait, il s'avère nécessaire d'analyser ces liens et les notions découlant du principe de responsabilité en droit de l'environnement. D'une part, il sera démontré que les principes de prévention et de précaution découlent du principe de responsabilité appliqué à la gouvernance. D'autre part, l'application du principe de responsabilité aux problématiques de gouvernance nécessite la définition des notions de provisoire et d'urgence et dans un contexte actuel d'impératifs économiques. Ce principe réorganise les rapports des secteurs privé et public.

\footnotetext{
1 Professora do Programa Stricto Sensu - Mestrado em Direito do IDP. Doutora e mestre em Direito pela Universidade de Limoges/ França - reconhecido pela Universidade Federal de Santa Catarina (UFSC). Professora do curso de pós-graduação lato sensu da ESPM, FIA e FMU. Professora convidada da Université Laval/Québec e professora visitante da Business School da Católica do Porto/UCP. Instituto IDP. Brasil. E-mail: glgarbaccio@hotmail.com

2 Professor Emérito da Universidade de Limoges, França. Diretor científico do Centro de Pesquisas Interdisciplinares em Direito Ambiental, de Ordenamento Territorial e Urbanístico da Universidade de Limoges, França. Presidente do Centro Internacional de Direito Ambiental Comparado, ONG internacional com status consultivo e acreditação permanente diante do Conselho Econômico e Social da ONU. Fundador da Sociedade Francesa para o Direito Ambiental, da Revista Europeia de Direito Ambiental e da Revista Jurídica do Meio Ambiente. Doutor honoris causa das universidades de Zaragoza (Espanha), de Bucareste (Romênia), de Sherbrooke (Canadá), de Santa Fe (Argentina). Oficial da Legião de Honra da França. Comandante da Ordem das Palmas Acadêmicas (França). Cavaleiro da Ordem Nacional do Mérito (França). Prêmio Elizabeth Haub (Universidade Livre de Bruxelas). Autor de inúmeros artigos e obras na área do Direito Ambiental. Serviços de consultoria especializada prestados a organismos internacionais. Université de Limoges. França. E-mail : michel.prieur@unilim.fr

${ }^{3}$ Coordinatrice et Professionnelle de recherche à la Chaire de recherche et d'innovation Goldcorp en droit des ressources naturelles et de l'énergie. Université Laval. Canadá. E-mail : glgarbaccio@hotmail.com

${ }^{4}$ Doutora e mestre em Direito pela Pontifícia Universidade Católica de SP. É advogada e professora da graduação e do Mestrado da Universidade Nove de Julho. Universidade Nove de Julho. Brasil. E-mail : baccio@hotmail.com
} 
Mots-clés : Gouvernance; Principes de responsabilité ; Principe de prévention ; Gestion de crise ; Principe de précaution; Provisoire ; Urgence.

Resumo: A governança, símbolo de uma nova modernidade nos modos de ação pública e gestão corporativa, constitui o quarto pilar do desenvolvimento sustentável. As ligações entre a governança e o princípio ambiental da responsabilidade parecem inextricáveis. Como resultado, é necessário analisar esses vínculos e noções decorrentes do princípio da responsabilidade em direito ambiental. Por um lado, será demonstrado que os princípios da prevenção e precaução derivam do princípio da prestação de contas aplicada à governança. Por outro lado, a aplicação do princípio da responsabilidade às questões de governança requer a definição das noções de temporária e de emergência e em um contexto atual de imperativos econômicos. Este princípio reorganiza as relações dos setores privado e público.

Palavras-chaves: Governança ; Princípio da responsabilidade ; Princípio da prevenção ; Gestão de crise ; Princípio da precaução ; Provisório ; Urgência.

\section{Abstract}

Governance, a symbol of a new modernity in modes of public action and corporate management, is the fourth pillar of sustainable development. The links between governance and the environmental principle of accountability seem inextricable. As a result, it is necessary to analyze these links and notions arising from the principle of responsibility in environmental law. On the one hand, it will be shown that the principles of prevention and precaution derive from the principle of accountability applied to governance. On the other hand, applying the principle of responsibility to governance issues requires defining the notions of temporary and emergency and in a current context of economic imperatives. This principle reorganizes private and public sector relations.

Keywords: Governance; Principle of responsibility; Prevention principle; Crisis management; Precautionary principle; Provisional; Urgency.

\section{Introduction}

La notion de gouvernance s'est imposée durant la dernière décennie du vingtième siècle comme le symbole d'une nouvelle modernité dans les modes d'action publique et dans le 
gouvernement des entreprises. Généralement entendue comme la démocratisation des processus de choix, elle constitue avec la prudence écologique, l'équité sociale et l'efficacité économique le " $4^{e}$ pilier du développement durable ».

L'environnement joue un rôle de "précurseur» dans la modernisation des formes de gouvernance mais cette volonté d'innovation s'accompagne du désir de renforcement des modes d'intervention traditionnelle de l'Etat - tel que "command and control». En effet, il s'agit principalement pour les responsables de l'environnement, de mettre en place une politique sectorielle bien définie, avec des dispositifs de contrôle classiques (réglementation, ressources budgétaires...) afin d'obtenir des résultats probants à court terme.

Par la suite, le débat sur la "gouvernance " s'est articulé autour de thèses opposées. La première stipule que la gouvernance est la seule solution adaptée aux problèmes actuels d'environnement, et elle est donc un parfait substitut aux politiques publiques traditionnelles et dépassées. Tandis que la seconde accuse la gouvernance d'être le problème car elle ne fait que renforcer l'impuissance collective face à des objectifs de plus en plus ingouvernables.

En 1995, W. SUNDERLIN, dans un article portant sur le changement global ${ }^{5}$, mettait déjà en évidence l'éclatement des réflexions des chercheurs autour des trois catégories " paradigmatiques » : d'un côté, ceux qui ont une vision essentiellement managériale de la " gouvernance "; de l'autre, ceux qui insistent sur les évolutions ou les différenciations culturelles; et enfin, ceux qui ont une vision " agonistique ", en terme de " rapports de force ", du problème, et considèrent que les solutions passent nécessairement par des changements structurels improbables (gouvernement mondial, leadership européen...).

II paraît urgent de sortir de l'impasse à laquelle aboutit ce débat. II nous faut bâtir un pont entre ces deux visions en nous plaçant dans une perspective de complémentarité entre Etat et société civile, entre démocratie représentative et démocratie délibérative ou participative, entre crédibilité institutionnelle et innovation managériale. En d'autres termes, mettre la gouvernance à l'épreuve de la « gouvernabilité ».

Cependant, toutes ces définitions n'apportent pas d'explication au développement - dans une perspective normative - connu sous le terme de "gouvernance " ces vingt dernières années. En réalité, ce phénomène s'explique par la globalisation et l'accroissement des échanges internationaux caractéristiques des années 80 mais également par la révolution qui a bouleversé les techniques de

\footnotetext{
${ }^{5}$ SUNDERLIN, W. D. Global environmental change, sociology and paradigm isolation. Global environmental
} change, vol. 5, number 3, Juin 1995. 
communication, par la perte de crédibilité de la régulation étatique et la fin de la bipolarisation mondiale...

Quand on utilise le mot gouvernance, on se réfère certainement à la définition beaucoup plus explicite, malgré les apparences, donnée par la Commission BRANDT sur la " gouvernance globale ", et reprise par BAIL au nom de la Commission Européenne : « la somme des voies et moyens à travers lesquels les individus et les institutions, publiques ou privées, gèrent leurs affaires communes. II s'agit d'un processus continu grâce auquel les divers intérêts en conflit peuvent être arbitrés et une action coopérative menée à bien. Ceci inclut les institutions formelles et les régimes chargés de mettre en application les décisions, ainsi que les arrangements que les gens ou les institutions ont acceptés ou perçoivent comme étant dans leur intérêt ${ }^{6} »$.

L'important dans cette définition c'est l'idée de processus interactif : une succession d'étapes à travers lesquelles de nombreux acteurs, qui n'ont pas le même intérêt et qui agissent à différentes échelles mais confrontés à un même problème, vont progressivement construire une représentation commune de cette réalité, lui donner un sens, se fixer des objectifs, adopter des solutions puis les mettre en œuvre collectivement sans que rien - ni cette représentation, ni ces objectifs, ni ce sens, ni cette interaction ne soient déterminés à l'avance?

On cherchera d'abord à éclaircir l'analyse de la gouvernance liée à l'environnement, notamment en ce qui concerne son interaction avec le principe de responsabilité. Puis on examinera l'application du principe de responsabilité dans les différents contextes de la gouvernance.

\section{Le développement durable et le principe de responsabilité : les notions qui en découlent}

Il apparaît que l'investissement selon des critères de développement durable a justement pour but de faire respecter et de promouvoir les biens collectifs et indivisibles. Le caractère financier de l'opération est critiqué, mais c'est sans doute un des moyens parmi les plus puissants pour réellement faire entrer ces préoccupations dans les faits, car le levier financier a l'avantage d'être uniforme et d'agir sur chaque entité (publique ou privée).

L’aspect répressif de la législation américaine - qui a notamment introduit la notion de responsabilité pénale de l'entreprise en 1991 - et le manque d'incitation à adopter des comportements responsables, pourraient trouver un contrepoids par ce biais.

\footnotetext{
${ }^{6}$ BAIL, Christophe. Environmental Governance : Reducing risks in democratic societies. Introduction paper, EEC, Future Studies Unit, 1996.

${ }^{7}$ MEUR-FEREC, Catherine. La GIZC à l'épreuve du terrain : premier enseignements d'une expérience française. www.developpementdurable.revues.org/index4471.html
} 
Cette solution reste cependant insatisfaisante, car cela signifie que le développement durable doit s'accommoder d'une certaine realpolitik économique et juridique dans la définition et l'application de ces critères. En effet, conformément à la tradition jurisprudentielle américaine, les juges sont contraints de limiter les effets négatifs des lois sans pour autant faire œuvre de législateur.

Nous allons souligner quelques aspects liés à la prévention et à la précaution, ainsi que l'association entre la prévention et la gestion de crise, car les changements climatiques vont générer d'autres crises à l'avenir.

La loi ne peut tout gérer. Elle se limite souvent à réparer ou à restaurer un équilibre. Dans le cas du développement durable, réparer est compliqué et difficile. La prévention devient dès lors un enjeu de taille, difficile à mettre en œuvre et, surtout, si cet enjeu n'est pas réellement en phase avec les préoccupations des autorités, plus souvent conditionnée par la gestion des urgences que par l'anticipation.

Dans un univers économique qui se veut de plus en plus incertain, tout progrès est perçu comme un facteur de risque. Ce conservatisme des esprits - qui se traduit par une défiance vis-à-vis de la technologie - tend, par le biais du principe de précaution, à renforcer la "judiciarisation » des sociétés. Ce principe peut, en réalité, être appliqué dans d'autres problématiques telles que celles de la protection de la santé humaine et de la sécurité alimentaire. Par conséquent, il y a consensus entre les spécialistes du sujet pour dire que le principe de précaution doit encore être amélioré.

Ce principe peut sembler, à premier abord, un résultat du simple bon sens, mais il n'en est rien. Il s'agit véritablement d'une révolution bouleversant le lien entre droit et science. En effet, le droit qui traditionnellement se limitait à s'opposer aux effets dommageables éventuels de l'occurrence de risques prouvés scientifiquement, intervient désormais dans l'anticipation de risques futurs, les risques climatiques par exemple encore mal appréhendés scientifiquement. Le principe de précaution ne stipule donc plus seulement l'adoption d'une approche prudente en cas de risques potentiels de dommages graves ou irréversibles, mais il postule également une gestion anticipatrice des risques globaux pouvant affecter la société.

En somme, les principes de prévention et de précaution imposent une réflexion profonde sur le droit et sur la façon dont celui-ci appréhende les avancées technologiques. Ils cherchent à susciter le débat social et à donner la parole tant aux experts qu'aux simples citoyens, par la mise en place de processus décisionnels qui restent largement à définir.

\section{I.1. L'association entre la prévention et la gestion de crise}


La gouvernance idéale implique un médiateur entre l'institution publique et la société civile. La confiance ne sera jamais parfaite. C'est une situation qui dure depuis longtemps. La vulnérabilité de la population liée aux changements climatiques amène à la dégradation de la confiance envers la production industrielle et envers les analyses scientifiques. L'effet de la pédagogie de la catastrophe constitue aussi une autre condition à laquelle la société est vulnérable : à cause de la non application du droit existant, la société exige une nouvelle loi, c'est-à-dire « la bonne loi », en espérant qu'elle soit « vraiment » appliquée le moment venu.

Les nouvelles politiques d'adaptation prétendent rendre supportables les conséquences des catastrophes liées aux changements climatiques. II s'agit des politiques d'atténuation, c'est-à-dire de réduction des risques. Mais il existe toujours le risque de l'émergence de nouvelles vulnérabilités. Les grands axes de la gestion sont : la prévention (l'action avant l'évènement) mais aussi l'action pendant et après l'évènement.

A partir de la prévention nous pourrons anticiper l'évaluation des dégâts de l'environnement. Il s'agit, d'abord d'une méthode monétaire. Le calcul doit être complété par la méthode délibérative avec la participation de la société civile.

C'est ici que nous pouvons rencontrer l'association entre la prévention et la gestion de crise. Nous devons suivre une des procédures de management : accompagner, prévenir et gérer la crise. La demande sociale évolue très fortement dans le sens d'une exigence croissante en matière de sécurité et rejette de plus en plus le concept de fatalité. L'idée que l'état des connaissances et des techniques doit permettre de maîtriser tous les dangers renforce cette exigence. En revanche, il n'est pas réaliste de penser pouvoir empêcher tous les événements nocifs pour l'environnement. La crise peut être déclenchée à partir d'un incident majeur ou tout simplement à partir d'un cas mineur. II faut préciser les risques de déclenchement d'une crise, déterminer les vulnérabilités et définir les mesures à prendre pour les réduire puis gérer la crise, lorsque l'aléa se réalise, avec l'objectif de minimiser l'impact de ces événements.

En l'absence d'une législation spéciale applicable, la gestion de la crise est encore plus compliquée. C'est dans cette perspective que la prévention occupe de plus en plus une place importante. Soucieux de ne pas provoquer une crise, dont le coût économique, social et moral pourrait être important, les gouvernements, les collectivités, les associations, les entreprises, etc. ont souvent comme démarche l'organisation de la prévention et de la gestion des crises. Leur objectif est de tout mettre en œuvre pour éviter les crises, notamment de confiance.

En étroite collaboration avec les services officiels et les autres opérateurs concernés, des cellules de crise peuvent être mises en place, si besoin. Celles-ci associeraient des professionnels 
concernés afin de définir les mesures à mettre en œuvre pour réduire autant que possible les effets de la crise et en traiter les causes.

Nonobstant le rôle fondamental de la prévention des crises, nous ne devons pas oublier les cas où nous n'avons pas encore des réponses définitives et fiables. C'est à ce moment que la précaution remplace la prévention.

\section{I.2. La précaution liée au développement durable}

Le principe de précaution peut être justifié, là où il est impératif de limiter, d'encadrer ou d'empêcher certaines actions potentiellement dangereuses sans attendre que le danger soit scientifiquement établi de façon certaine. Il s'agit donc bien d'un principe de décision en l'absence de certitudes scientifiquement établies. Il est mis en application dans la convention de Vienne sur la protection de la couche d'ozone de 1985, et devient principe fondateur du droit de l'environnement avec le rapport Brundtland ${ }^{8}$, sur le développement durable, en 1988. II entre dans le droit positif européen avec l'article 130 du Traité de Maastricht ${ }^{9}$. En droit positif français, selon l'article $1^{\text {er }}$ de la loi Barnier: "Le principe de précaution selon lequel l'absence de certitudes, compte tenu des connaissances scientifiques et techniques du moment, ne doit pas retarder l'adoption de mesures effectives et proportionnées visant à prévenir un risque de dommages graves et irréversibles à l'environnement, à un coût économiquement acceptable ${ }^{10}$. GODARD distingue deux versions du principe de précaution. Une version "faible ", où l'idée de précaution représente un critère partiel qui, pour avoir un effet pratique, doit être complétée par d'autres éléments de justification. Une version «forte » où l'idée de précaution est alors « un critère absolu » destiné à se substituer à toute autre exigence de signification ${ }^{11} »$.

L'aspect économique est surtout dominant dans la formulation française du principe de précaution. Le texte de la loi invite donc à une comparaison des coûts et des avantages attendus des mesures de protection envisagées, et conduit à fixer des critères de décision.

Les experts ont fait un calcul beaucoup plus directement politique, car ils étaient persuadés que la transparence et le contrôle constituent en fait le prix à payer pour éviter que ne se développe dans la population une "peur irrationnelle » du génie génétique. En acceptant le système de

\footnotetext{
8 "Notre avenir à tous », 1988.

${ }^{9}$ Devenu l'article 191 du traité de Lisbonne sur le fonctionnement de l'Union européenne signé en 2007.

${ }^{10}$ Loi n 95-101 du 2 février 1995, relative au renforcement de la protection de l'environnement (art. L. 110-1-II$1^{\circ}$ du code de l'environnement.

${ }^{11}$ GODARD, Olivier. L'ambivalence de la précaution et la transformation des rapports entre science et décision. p. 19.
} 
l'autorisation préalable, les scientifiques et les industriels donnaient ainsi un gage de bonne foi à une opinion publique incrédule et méfiante. Il s'agissait sans doute de "prévenir les risques " mais, davantage de « conjuguer les craintes».

C'est dans les conflits et les pressions qu'elles font peser les unes sur les autres que peuvent se dégager des solutions positives : c'est une conception plus conflictuelle, moins " raisonnable », de la démocratie. Cela conduit HERMITTE ${ }^{12}$ à défendre une version moins réductrice et moins partielle, du principe de précaution que GODARD. Elle insiste sur les modifications que son application introduit tant dans la "culture du risque » que dans le statut juridique de l'innovation technologique. Du principe libéral selon lequel " tout ce qui n'est pas défendu est permis ", on est passé au régime du privilège, de l'autorisation préalable, du tout ce qui n'est pas permis est défendu. Cela ne revient pas à s'abstenir d'agir, mais à prendre les mesures nécessaires pour réduire un risque suspecté.

Le principe ne s'arrête pas à l'interdiction, il introduit une dynamique qui permet d'en sortir : celle des démarches aboutissant aux autorisations d'incitation à développer les connaissances. C'est à cela que vise l'inversion de la charge de la preuve.

La prudence, et son bricolage empirique, sont ainsi ouverts sur l'audace et l'innovation. C'est de ce côté que se trouvent les lumières, que se trouvent ceux qui " osent savoir ". C'est une bonne façon d'envisager l'avenir.

Le principe de précaution pour certains penseurs, serait source d'importants coûts pour la collectivité car il amènerait au blocage de l'initiative économique et de toute innovation scientifique et technologique.

Tout d'abord, il serait fallacieux de voir dans le principe de précaution un mécanisme antiscience » alors que celui-ci vise le redoublement de l'effort de recherche. Ensuite, celui-ci ne constitue guère un obstacle à l'innovation technologique, il peut tout au plus retarder la mise sur le marché de celle-ci. Il oblige ainsi à la révision du contenu d'une partie des efforts de recherche et de développement.

Ce principe ne peut donc être mis en œuvre que sous certaines conditions de fond et de forme. Le risque qu'un dommage se produise ne doit pas être un simple fantasme, une simple hypothèse non étayée, il doit être "suffisamment documenté " par des indications scientifiques fiables et solides au regard des évaluations scientifiques disponibles. Une pure élucubration n'autorise donc pas à le mettre en œuvre.

\footnotetext{
${ }^{12}$ HERMITTE, Marie-Angèle cité par LARRERE, Catherine et LARRERE, Raphaël. Du bon usage de la nature. Pour une philosophie de l'environnement. Alto Aubier, Paris, 1997 (mars). p.141.
} 
Enfin, il ne s'applique, exclusivement, qu'à la condition que les hypothèses scientifiques soient suffisantes. Deux autres conditions d'applicabilité doivent également être respectées. Premièrement, toute mesure de précaution doit être proportionnée au risque redouté, ce qui signifie que les moyens choisis doivent être limités à ce qui est effectivement nécessaire pour assurer la sauvegarde de la santé publique et/ou de l'environnement. En second lieu, quelle que soit la mesure de précaution retenue, celle-ci doit pouvoir être soumise à un réexamen périodique au vu des connaissances et données scientifiques nouvellement acquises.

Le principe de précaution n'est ni un principe d'abstention ni un principe qui, structurellement, contraindrait le décideur à adopter la décision la plus sécuritaire. Reprenant l'annonciation de la Cour de Justice des Communautés Européennes (CJCE), le Comité de la prévention de la prévention et de la précaution relatif au principe de précaution écrit : «la subordination du maintien de l'autorisation d'une substance à la preuve de l'absence de tout risque même purement hypothétique serait à la fois irréaliste - dans la mesure où une telle preuve est en règle générale impossible à fournir du point de vue scientifique, dès lors qu' un niveau de risque zéro n'existe pas en pratique - et contraire au principe de proportionnalité ${ }^{13} »$.

"Le développement est semblable à une étoile morte dont on perçoit encore la lumière, même si elle s'est éteinte depuis longtemps, et pour toujours ${ }^{14}$ ».

Le débat ne doit pas se concentrer sur plus de développement économique ou moins de développement économique, mais sur un développement durable et un développement humain.

Issu du Rapport Brundtland, ce principe était déjà implicite dans les principes 5 et 8 de Stockholm 1972, et aussi dans les principes 3 et 4 de Rio1992.

La précaution comporte deux éléments fondamentaux : l'incertitude et l'irréversibilité. Elle est une action anticipatrice et $a$ priori qui est préférée aux mesures a posteriori, telle la réparation, la restauration ou la répression par exemple.

Quatre instruments durables contribuent à sa mise en œuvre : l'étude d'impact (l'obligation de prendre en compte l'environnement avant l'action), l'autorisation préalable des activités polluantes, la lutte à la source pour les biens et produits et les éco-audits.

Le premier instrument est en même temps une procédure administrative que l'on peut qualifier de révolutionnaire car elle va pénétrer dans l'ensemble du dispositif de droit administratif et contraindre les autorités publiques et les acteurs privés à changer d'attitude. Le deuxième instrument, l'autorisation préalable est exigée pour l'exercice de toute activité polluante ou risquant de porter

\footnotetext{
${ }^{13}$ CJCE cité par l'Avis du Comité de la prévention et de la précaution relatif au principe de précaution, 2004. p.7 ${ }^{14}$ RIST, Gilbert. Le développement. Histoire d'une croyance occidentale. Presses de Sciences Po, Paris, 1996, p. 377.
} 
atteinte à l'environnement. Par le troisième instrument, la gestion écologiquement rationnelle prônée par le Programme des Nations Unies pour l'environnement (PNUE) exige, au lieu de réduire les rejets et les émissions de polluants, de s'attaquer à la source de la pollution en imposant une conception et une fabrication des machines et des produits qui n'engendrent pas ou très peu de nuisances lorsqu'on les fait fonctionner ou lorsqu'on les consomme (produits, recyclables, véhicules moins polluants, etc.). Il s'agit du principe 8 de la Déclaration de Rio de 1992. Le quatrième et dernier instrument, les écoaudits introduisent un système facultatif d'audit d'environnement qui incite fortement à améliorer la gestion des entreprises (plans verts par exemple).

On peut dès lors formuler un modèle théorique de développement durable. Cette représentation est la plus connue et la plus divulguée par les moyens de communication.

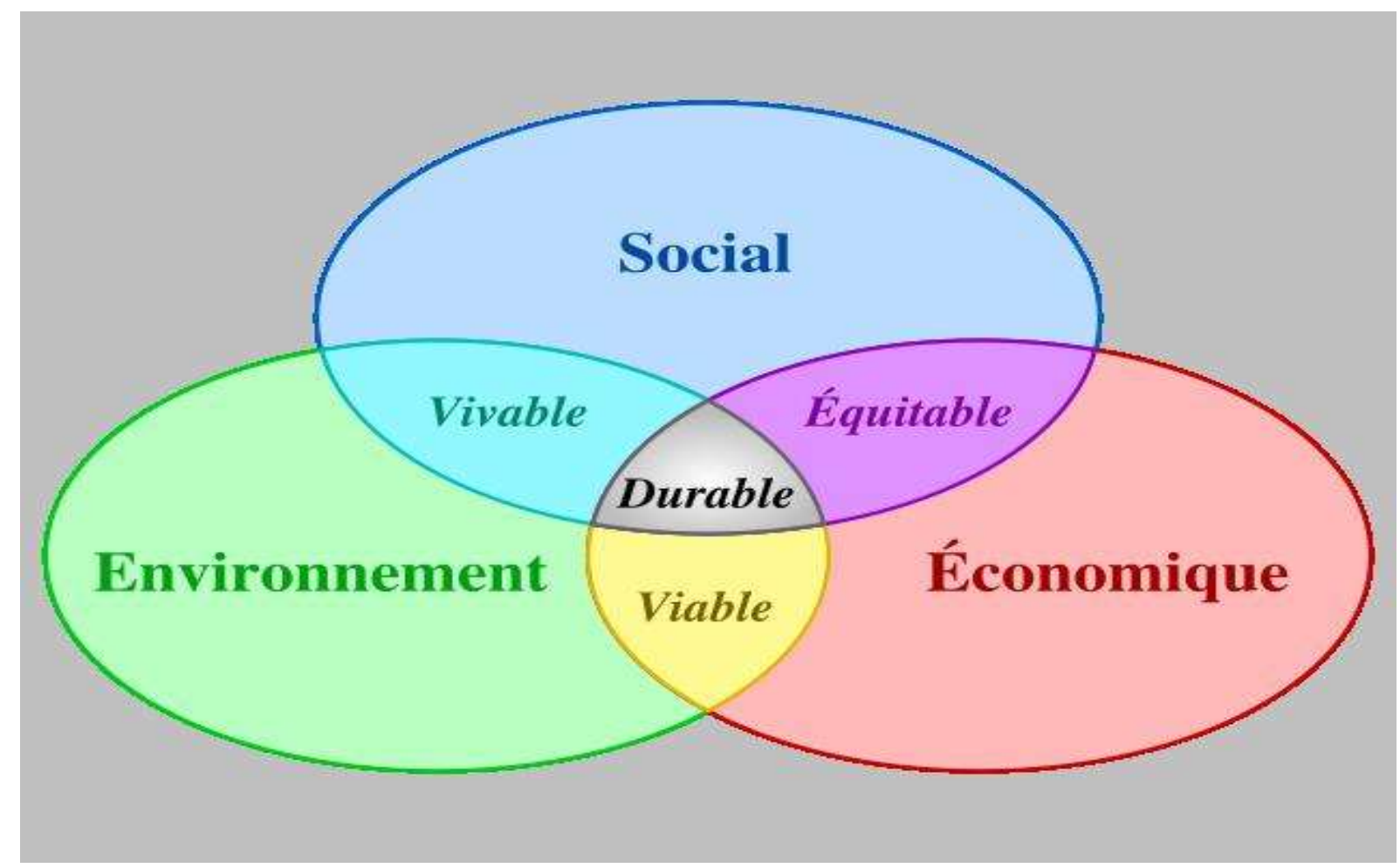

L'évolution des recherches et des débats sur la prise en compte des aspects social, économique et environnemental, nous amène à considérer la représentation suivante du développement durable comme le modèle plus adéquat : 


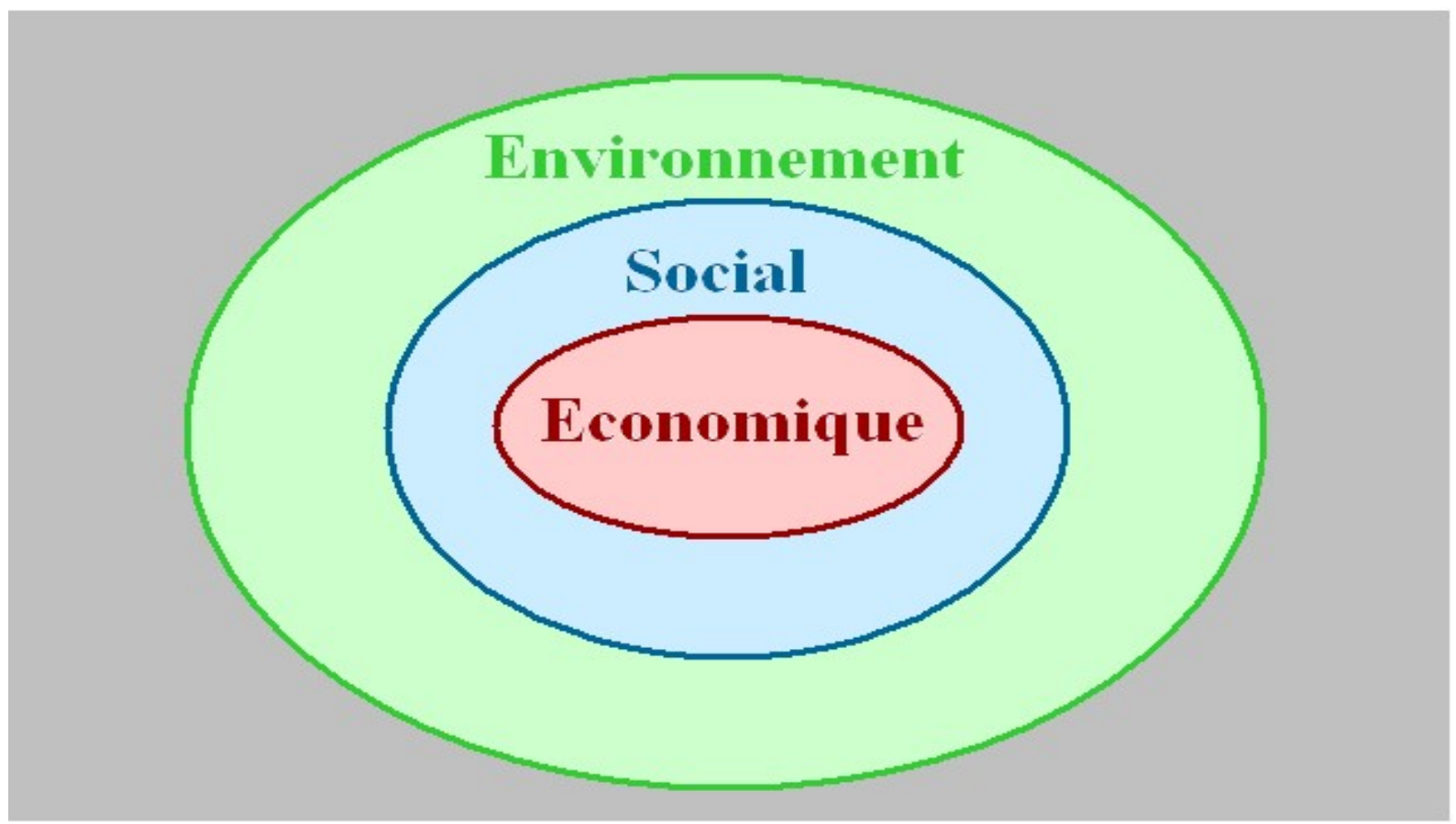

Nous partons de l'idée suivante : c'est mieux d'arriver à un résultat approximativement correct qu'à un résultat totalement incorrect.

Le développement durable se fonde sur trois axes indissociables que sont la performance économique, le progrès social et la préservation de l'environnement. II s'engage également sur la durée et dans un contexte de démocratie.

Traités jusque-là de façon isolée, les problèmes rencontrés par l'homme sont désormais abordés dans leur globalité par le développement durable. Ce bouleversement dans les relations entre I'homme, le temps et l'espace, tenant compte de la problématique du « ici et maintenant " allier à celle du « là-bas et demain », justifie nos choix de politiques.

Un des principes proposés dans le rapport Brundtland est « qu'il faut penser globalement et agir localement ». 


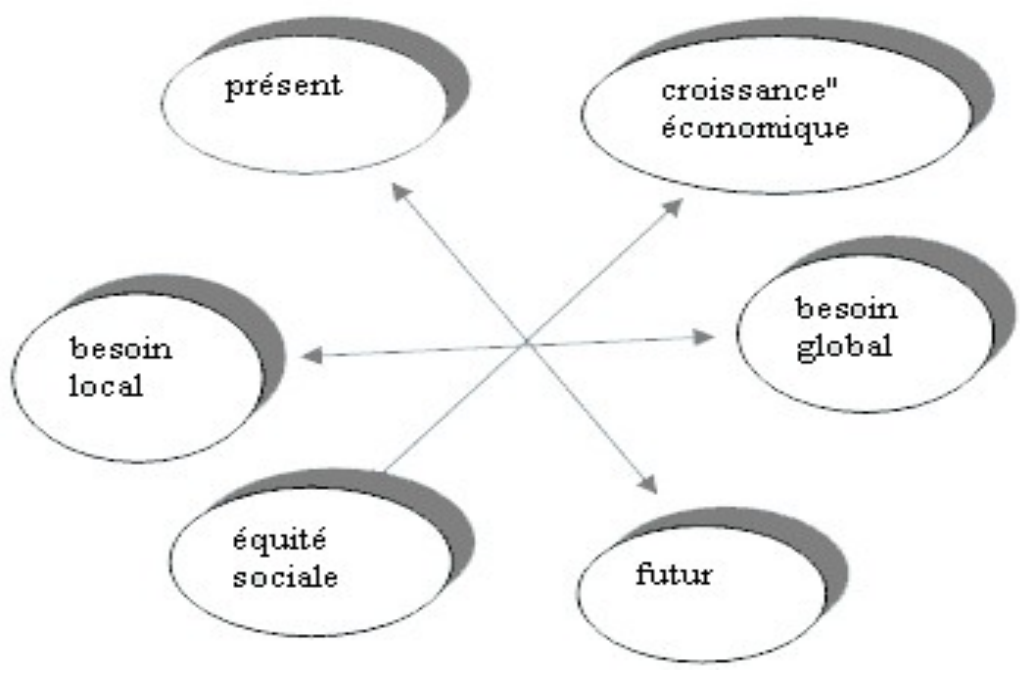

Source : http://www.notre-planete.info/ecologie/devdurable/devdurable 1.php

Le défi, dans la construction du principe de précaution, c'est de pouvoir intégrer des besoins contradictoires nés, d'une part, de la nécessité de protéger le droit à la libre entreprise, ainsi que l'esprit d'innovation si indispensable au progrès technologique pour le développement économique, et d'autre part, la nécessité de réduire le risque d'effets négatifs sérieux et irréversibles sur l'environnement.

Les travaux économiques sur la responsabilité civile proviennent du prolongement de la théorie économique des accidents. La notion de responsabilité civile (pour faute et sans faute) ${ }^{15}$ remplit une fonction préventive et indemnitaire. L'anticipation de la sanction financière incite les agents économiques, les entreprises en particulier, à adopter ex-ante un comportement de gestion du risque.

La théorie économique montre qu'un tel instrument est efficace : quelle que soit la règle de responsabilité retenue (pour faute ou sans faute), celle-ci conduit les agents économiques à choisir un niveau de précaution optimal. La règle de responsabilité pour faute motive l'évitement de la faute ; la règle de responsabilité sans faute incite à prendre des mesures qui minimisent le coût prévu de l'accident.

La règle de la responsabilité étendue suppose que l'on reporte en partie le risque sur un partenaire de l'entreprise (une banque ou une assurance), qui détient un contrôle, direct ou indirect

\footnotetext{
${ }^{15}$ On parle de responsabilité sans faute lorsque l'agent économique imposant les risques est légalement responsable des dommages qu'il provoque, quel que soit par ailleurs le degré de précaution avec lequel il exerce son activité. On parle de responsabilité pour faute lorsque l'agent économique imposant les risques est légalement responsable des dommages qu'il provoque si son effort de prévention est inadéquat par rapport à une norme ou standard de comportement fixé par le tribunal compte tenu des caractéristiques de la situation (B. Deffains, 1999).
} 
(financement de l'activité, prime d'assurance), sur celle-ci. La Convention de Lugano ${ }^{16}$ met ainsi « à la charge des banque un risque "vert » en invoquant le fait qu'elles prennent souvent une part active dans les décisions des entreprises qu'elles financent ». Une autre solution consiste à contourner le problème de la solvabilité par l'assurance. Dans les deux cas, l'entreprise partage la gestion du risque avec un organisme extérieur, qui ne détient pas toute l'information sur le processus de production de l'entreprise et sur les probabilités d'accident. Le transfert de risque peut alors être plus efficace vers la banque que vers l'assurance. Les assurances peuvent toutefois élargir leurs marges de manœuvre en faisant appel aux mécanismes de réassurance.

Toutefois, tous ces mécanismes peuvent être ignorés lorsque la menace de sanction n'est pas crédible, par insuffisance de contrôle ou par absence de solvabilité. L'extension du principe de responsabilité permettrait de dépasser cette limite.

Le principe de précaution a connu une première application juridique d'importance à l'occasion de la définition des mesures de protection de la couche d'ozone stratosphérique ${ }^{17}$. Puis, d'autres textes sont venus renforcer le poids du principe de précaution. L'article 3, alinéa 3 de la Convention Cadre des Nations Unies sur les Changements Climatiques - CCNUCC - du 9 mai 1992, entrée en vigueur le 21 mars 1994 indique qu'il « incombe aux Parties de prendre des mesures de précaution pour prévoir, prévenir ou atténuer les causes des changements climatiques (...). Quand il y a risque de perturbations graves ou irréversibles, l'absence de certitude scientifique absolue ne doit pas servir de prétexte pour différer l'adoption de telles mesures (...) ". Le traité d'Amsterdam sur I'Union européenne du 2 octobre 1997 précise de son côté, dans son article 174, alinéa 2, que la politique de la Communauté dans le domaine de l'environnement « est fondée sur les principes de précaution et d'action préventive (...) ${ }^{18}$.

La prévention peut être mise en place chaque fois que la distribution de probabilité est connue; lorsque celle-ci n'est pas fixée, la précaution s'impose comme attitude de prudence autorisant la prise de décision en attente d'informations supplémentaires sur le phénomène que l'on veut gérer.

L'approche économique du principe de précaution indique que la perspective de recevoir de l'information ne justifie pas le report de la prise de décision; des décisions peuvent être prises, qui concilient une action sur la dynamique de la pollution et sur la dynamique de l'accumulation des connaissances. Dans le cas de l'effet de serre, les mesures d'économie d'énergie limitent les émissions

\footnotetext{
${ }^{16}$ Convention du Conseil de l'Europe sur « la responsabilité civile des dommages résultant d'activités dangereuses pour l'environnement », 21 juin 1993. (non entrée en vigueur).

${ }^{17}$ Convention de Vienne du 22 mars 1985, puis Protocole de Montréal en septembre 1987.

${ }^{18}$ Repris en 2007 à l'art. 191-2 du traité de Lisbonne.
} 
de carbone à un coût macro-économique qui peut être nul ou négatif, et inscrivent donc l'économie sur une trajectoire qui préserve les possibilités d'actions futures.

Un taux d'actualisation élevé écrase le long terme, ce qui peut être perçu comme une injustice vis-à-vis des générations futures, surtout lorsque celles-ci auront à subir des dommages attribuables aux modes de vie des générations actuelles. Le recours à un taux d'actualisation positif se justifierait alors par deux arguments principaux. D'une part les agents économiques manifestent une préférence pour le présent, préfèrent recevoir un euro aujourd'hui plutôt que demain, ce qui signifie qu'ils valorisent moins les sommes à venir que les sommes présentes. D'autres part, l'histoire économique de l'après Seconde guerre mondiale le montre avec éclat, les générations futures bénéficieront probablement d'un niveau de vie bien supérieur à celui des générations actuelles.

Pour garantir effectivement aux générations futures ce niveau de vie, nous devons anticiper l'analyse de certains contextes qui, en ce moment, peuvent être considérés comme exagérés, mais qui gardent toujours un sens d'équilibre et de cohérence.

\section{Le principe de responsabilité appliqué aux différents contextes de la gouvernance}

La réforme des institutions internationales pose cinq questions :

A - Quel Etat ? Aujourd'hui I'Etat doit être responsable. Cette responsabilité est double: responsabilité classique de tout pouvoir démocratique face au peuple qui l'a élu; responsabilité « nouvelle » face à la communauté internationale, nombre d'événements dits intérieurs (par exemple catastrophe écologique) ayant des répercussions extérieures, en particulier par le biais des flux (flux de réfugiés, flux de déchets, .....). L'Etat doit être « normal » avec un double rôle : faire prendre en considération les préoccupations de sa population, de son territoire auprès de l'extérieur ; incorporer les normes venues de l'extérieur dans l'espace dont il a la charge.

$\mathrm{B}-$ Quelle régulation ?

C-Quelle solidarité ? Deux difficultés dominent : équilibre et responsabilité. La solidarité doit s'accompagner d'une responsabilisation des bénéficiaires (conformément à l'adage bien connu de Mao Zedong : « si vous donnez à quelqu'un qui a faim un poisson, il mangera une fois ; si vous apprenez à pêcher à cette personne, elle pourra se nourrir toute sa vie. "). D’où la notion de conditionnalité : toute aide doit s'inscrire dans une relation contractuelle, le bénéficiaire de l'aide s'engage à satisfaire certaines obligations (respect de règles, réalisation de résultats). Une solidarité illimitée 
transformerait tous les hommes en assistés. Il faut de critères. Ici apparaît l'idée - bien difficile à mettre en œuvre - d'urgence : la catastrophe doit être évidente, massive et porteuse de dégâts irréparables.

D - Quelle participation politique ? L'Etat, parce qu'il est encore la seule machine transformant l'être de nature qu'est l'homme en citoyen porteur de droits, demeure le lieu de la légitimité politique. En outre, avec le temps, ces phénomènes, suscités par l'éthique de conviction de (Max Weber), découvrent l'éthique de responsabilité. La vie démocratique n'est plus le monopole des Etats Nations.

E-Quel policier ? L'ONU se construit autour d'un policier institutionnel, le Conseil de sécurité. Or ce policier n'agit que si ses membres sont d'accord (au moins neuf voix sur quinze, aucun veto des cinq permanents). Et l'ONU ne prive pas les Etats du droit de légitime défense.

Bien que la responsabilité des émissions de GES incombe à trois secteurs bien identifiés, les transports, le résidentiel et l'industrie, nous nous contenterons dans le cadre de cet article d'examiner plus particulièrement quels instruments pouvent être utilisés pour parvenir à des réductions d'émissions dans le secteur industriel. Chaque secteur a de réelles spécificités, et il conviendra sans aucun doute d'adopter des solutions différenciées pour chacun d'entre eux.

\section{II.1. Les notions de provisoire et d'urgence}

Même si la notion de provisoire a évolué et que l’on admet en principe que le juge des référés peut, pour justifier sa décision, examiner le fond du droit, il n'en reste pas moins que prétendre que la notion de "provisoire " ne concerne plus que l'inopposabilité de la décision au juge du fond, est nettement exagérée. Si dans l'aménagement de situations d'attente ou dans l'octroi de provisions le juge des référés peut aller jusqu'à causer à la partie à charge de laquelle il ordonne la mesure de certains inconvénients, voire de certains dommages, il ne peut jamais aller jusqu'à infliger, sous prétexte qu'une des parties est menacée d'un préjudice, à l'autre une situation irréversible.

Il en découle que le juge des référés ne peut ordonner des mesures irréversibles qu'avec une extrême circonspection et que dans la mesure où il existe une évidence suffisante de bon droit, il doit rester prudent dans l'appréciation du caractère grave et éventuellement irréparable du dommage dont le demandeur se dit menacé... en ne perdant pas de vue que dans l'optique du code civil toute obligation de faire ou de ne pas faire se résout en dommages intérêts.

L'enthousiasme dont certains font preuve à l'égard du référé, juridiction où, en raison de l'urgence même, la demande ne peut faire l'objet que d'un examen sommaire et succinct, fait craindre la naissance d'une justice sommaire et tout aussi peu nuancée que celle d'un « far-west » légendaire où l'on pendait rapidement et où l'on vérifiait ultérieurement la culpabilité. 
L'urgence a toujours été assez délicate à appréhender. C'est pourquoi, elle connaît de multiples déclinaisons dans l'ensemble des branches du droit et donne lieu à des études aussi variées.

C'est bel et bien au sein de la théorie de la dérogation qu'est très largement enchâssée la notion d'urgence qui, de manière classique, comprend deux pôles structurels : elle naît d'une situation matérielle, et génère une réaction décisionnelle. Elle naît d'une situation particulière imposant que soient prises des décisions particulières.

A cet égard, certaines similitudes théoriques existent entre l'urgence et d'autres notions juridiques tels que :

les circonstances exceptionnelles, avec notamment l'article L 425-3 du Code de l'environnement. Et ce du fait qu'elles font référence à des circonstances anormales ou exorbitantes et peuvent ainsi participer à la caractérisation d'une urgence ;

la force majeure, notamment les articles R 214-33 et R 236-16 du Code de l'environnement, de manière plus éloignée et ce à travers leur caractère exceptionnel commun ; ou encore la nécessité, notamment les articles L 222-1, L 331-5, L 411-1 ou L 541-10 du même code, en ce que la fonction dérogatoire de l'urgence l'intègre. En effet, c'est bien la nécessité - plus d'efficacité - qui nous pousse à déroger au droit commun.

C'est d'ailleurs avec l'idée de " nécessité " qu'on peut théoriquement rapprocher le plus le concept « d'urgence ». L'essentiel des caractères de la nécessité porte sur l'intérêt à protéger, tandis que l'essentiel des caractères de l'urgence porte sur l'action dans le temps; action qui par elle-même représente un intérêt en soi qu'il importe de sauvegarder. Mais limiter la notion d'urgence à agir vite n'a pas de sens en soi. II n'a de sens qu'au regard de ce qu'il s'agit de permettre, de protéger, d'interdire, etc. Et plus précisément, la rapidité d'une action ne se justifie qu'en égard à l'imminence certes, mais également à l'importance de l'objet visé (autorisation, préservation, interdiction, etc.). Réduire l'essence de l'urgence à la seule dimension temporelle est de ce point de vue problématique.

Ce que nous amène à étudier les deux éléments constitutifs de l'urgence : la temporalité et la gravité particulière (imminence du péril).

La distinction entre l'urgence réparatrice et l'urgence préventive est classique ${ }^{19}$. La différence existante entre ces deux déclinaisons tient bien entendu à la temporalité de l'action administrative et à son positionnement par rapport à la survenance de l'évènement nocif. La première renvoie à des actions en amont, destinées à prévenir la survenance dudit évènement, la seconde à des actions $a$ posteriori dont l'objet est de limiter au plus vite les conséquences d'un évènement déclencheur qui a

\footnotetext{
${ }^{19}$ Loi ${ }^{\circ}$ 2000-597 (J.O. du $1^{\text {er }}$ juillet 2000) relative au référé devant les juridictions administratives.
} 
d’ores et déjà eu lieu. S'agissant de la distinction matérielle du préventif et du curatif, l'on perçoit qu'elle s'incarne ici dans cette double hypothèse. L'urgence est, dans les deux cas, motivée par la dangerosité du dépassement du seuil pour la santé humaine et pour l'environnement, dangerosité scientifiquement établie et posée comme hypothèse pour le juriste.

Il faut qu'il y ait urgence, c'est-à-dire péril en la demeure. Les mesures doivent rester provisoires. La notion de provisoire ne se réduit ni au conservatoire, interdisant de trancher le fond du litige, ni au temporaire, imposant au juge de limiter les effets des mesures dans le temps. Ces mesures sont provisoires en ce sens qu'elles peuvent être modifiées ou rétractées par le même juge lorsque le changement de circonstances le justifie.

La précaution traitée ci-dessus est liée à la notion de provisoire. L'urgence est un concept divfférent qui est plutôt lié à la notion de prise de décision rapide. L'urgence est temporaire mais aussi substantielle.

La volonté de la Déclaration des droits de l'homme ${ }^{20}$ était d'affirmer que le respect de la vie ne doit pas être perçu comme un simple « réflexe » face aux risques liés à l'utilisation des nouvelles technologies. Le recours aux droits de l'homme et le rééquilibrage des pouvoirs de la science au profit des patients et des citoyens est, bien sûr, un élément important pour « préserver la dignité de la personne et le respect universel et effectif des droits de l'homme et des libertés fondamentales ${ }^{21}$ ».Mais, la vision du respect de la vie va au-delà : il s'agit de prendre en compte «l'influence de plus en plus (grande) que les progrès rapides des sciences et des technologies ont sur l'idée que nous avons de la vie et la vie elle-même ${ }^{22}$ ».

C'est non seulement ce que nous sommes, mais la conscience que nous en avons et l'organisation sociale qui en découle, qui est un jeu. Dès lors il y a urgence - mais une urgence existentielle - pour « la communauté internationale d'énoncer des principes universels sur la base desquels l'humanité pourra répondre aux dilemmes et controverses de plus en plus nombreux que la science et la technologie suscitent pour l'humanité et l'environnement ${ }^{23}$ ".

Si le respect de la vie sous toutes ses formes doit guider l'action de l'homme au regard de la mise en œuvre des sciences et des technologies, cette référence permanente de la Déclaration s'inscrit dans une démarche dynamique, " consciente de la capacité propre aux êtres humains de réfléchir à leur existence et à leur environnement pour éviter le danger et assumer (leurs) responsabilités ${ }^{24}$ ». II

\footnotetext{
20 Unesco, Déclaration universelle sur la bioéthique et les droits de l'homme, 19 octobre 2005.

${ }^{21}$ Unesco, Déclaration universelle sur la bioéthique et les droits de l'homme, préambule, $3^{\mathrm{e}}$ consid., 19 octobre 2005.

${ }^{22}$ Op. cit. $2^{\mathrm{e}}$ consid.

${ }^{23}$ Op. cit. $4^{\mathrm{e}}$ consid.

${ }^{24}$ Op. cit. $1^{\mathrm{er}}$ consid.
} 
ne s'agit pas d'une approche purement " conservatrice " visant à figer la vie humaine et les autres formes de vie mais il s'agit, au contraire, de souligner la nécessaire quête d'un équilibre entre "les progrès des sciences et technologies, fondés sur la liberté de la science et de la recherche, et la promotion du bien-être des individus, des familles, des groupes ou communautés et de l'humanité dans son ensemble ${ }^{25} »$.

Nous ne pouvons pas laisser de côté le contexte économique qui nous soumet à certaines responsabilités, soit au niveau local, soit au niveau global, soit au niveau public, soit au niveau privé.

\section{II.2. La mise en place de la responsabilité dans un contexte d'impératifs économiques}

L'actualité liée à la globalisation des activités économiques dément la pertinence de ce compartimentage. L'écran de l'action privée - fréquemment celle du private attorney general incité par la perspective de dommages intérêts punitifs à poursuivre des infractions au droit économique pour le compte des autorités publiques - dissimule mal l'implication directe des Etats dans le contentieux international. En effet, l'introduction massive du droit public économique dans le champ des conflits de lois issus de l'interconnexion des marchés s'accompagne d'une politisation caractérisée des litiges.

Or, la multiplication spectaculaire du nombre de régulations économiques dans le monde incite aujourd'hui à réfléchir aux moyens permettant d'en assurer l'articulation et d'atténuer ainsi l'état anarchique de l'ordre économique international de droit privé. Sans doute, faut-il voir désormais dans cette tension, qui réaménage les rapports du privé et du public, la nouvelle économie politique du droit international privé issue de la globalisation des marchés.

Les quelques tentatives d'identifier plus concrètement l'Etat pourvu du meilleur avantage de régulation - " best regulatory advantage » -, s'étant soldées par un curieux retour aux solutions du premier "Restatement ", jugées plus sûres, l'attention de la doctrine économique s'est détournée pour l'essentiel de la recherche de la loi la meilleure - " the best fit » - pour découvrir les vertus de l'autonomie de la volonté, étendue du domaine des contrats à celui des délits liés au marché.

La transformation progressive des conflits de lois dans l'Union européenne sous l'influence du marché intérieur fait lentement prendre conscience que le règlement des conflits de lois peut représenter un enjeu économique important, au moins dans ce contexte quasi fédéral. Ainsi, l'apparition de nouveaux mécanismes de répartition des compétences étatiques en matière de droit

${ }^{25}$ Op. cit. $12^{\mathrm{e}}$ consid. 
public atteste les transformations profondes qui accompagnent nécessairement l'étiolement du paradigme privatiste.

Le troisième "Restatement » des relations externes des Etats-Unis a tenté d'introduire la "quête du raisonnable " sous la forme d'une auto-discipline dans l'exercice par les juridictions fédérales de l'impérialiste juridiction américaine en matière économique. Dans tous ces cas, on relève une ouverture au droit public étranger - que ce soit par la prise en considération des politiques étrangères ou par l'instauration spontanée de mesures de coopération entre les autorités - fondée sur le constat de l'interconnexion des droits économiques. La globalisation apparaît désormais comme un fait qui exige de surmonter le "tabou » traditionnel du droit public étranger - un peu comme l'accélération des moyens de transports et de communication avait imposé aux juges de surmonter le tabou qui, à la fin du XIXème siècle, affectait encore l'application du droit étranger. On peut même estimer qu'en ouvrant leurs marchés et en acceptant l'interconnexion, les Etats renoncent au monopole traditionnel du droit public économique du for devant leurs propres autorités.

Actuellement, l'analyse économique des conflits de lois s'est orientée en faveur d'une augmentation de la part de l'autonomie de la volonté dans le règlement de ces conflits. Les fondements de ces derniers sont des analyses fondées sur la souveraineté et les intérêts gouvernementaux. Ceux-ci se sont vus rejeté en faveur d'une méthodologie fondée sur la recherche de l'efficience et l'on redécouvre les vertus du libre choix des parties.

Un autre courant est allé plus loin encore dans la « re-privatisation » du droit international privé. Il repose sur la conviction des bienfaits de la concurrence entre les différents législateurs locaux - empruntée à l'analyse économique du fédéralisme -. L'exercice par les parties de leur faculté de libre choix mettrait précisément ceux-ci en compétition.

Une illusion cependant est celle des vertus régulatrices du marché : les lois plus laxistes moins protectrices pour les actionnaires et moins coûteuses - seraient offertes aux opérateurs prêts à prendre des risques, tandis que les investisseurs plus prudents - « risk-averse » - pourraient disposer d'un éventail de régimes offrant une protection accrue, à un prix bien entendu supérieur. Ainsi, en matière de régulation boursière, Stephen $\mathrm{CHO}$ et Andrew GUZMAN se proposent d'approfondir ce thème, en y intégrant des conclusions tirées de l'observation du principe de reconnaissance mutuelle telle qu'il est pratiqué dans ce même domaine dans le cadre de diverses conventions bilatérales, notamment entre les Etats-Unis et le Canada, chaque Etat acceptant de tenir la législation de l'autre comme équivalente à la sienne propre dans le cas d'opération transfrontières intéressant les deux pays.

La proposition des auteurs consiste à généraliser cette réciprocité sur le plan global : la société émettrice de valeurs en bourse choisirait librement la législation devant régir l'émission, laquelle serait 
ensuite reconnue par l'ensemble des autorités de marchés de divers lieux où des opérations interviendraient par la suite sur les titres émis. Il s'agirait donc d'une « reconnaissance portable ", car reposant sur la dissociation entre la loi applicable et le lieu des opérations ; la loi initialement choisie accompagnerait les valeurs émises dans tous leurs "déplacements " ultérieurs. Le résultat, selon les auteurs, ne serait pas une diminution uniforme de la protection des investisseurs, mais plutôt une spécialisation législative, faisant apparaître sur l'échiquier global des offres de législation plus ou moins protectrices, qui attireraient ainsi des catégories différentes d'opérateurs en fonction de préférences très variables. Le marché global deviendrait plus efficient, maximisant les bienfaits de la compétition ; pour le marché américain, le bénéfice serait également incontestable, neutralisant l'effet dissuasif qu'exerce à l'heure actuelle le contenu excessivement rigoureux de la loi fédérale sur les investisseurs étrangers.

Indéniablement séduisante, cette thèse "de dérégulation " semble appelée à exercer une certaine influence au sein de l'Union européenne. Cependant, en tant qu'elle postule la fongibilité absolue des législations, l'idée de "réciprocité portable " est difficile à transposer au marché global, en l'absence de communauté juridique faite de régulations économiques homogènes. En effet, selon le modèle de compétition pour les biens publics de Tiebout, dont dérive la théorie économique du fédéralisme, les conditions requises pour la compétition législative sans distorsion, résultent du choix éclairé des parties et de l'absence d'externalités, c'est-à-dire, d'effets néfastes dont le coût serait supporté par les tiers. Or, précisément, dans un domaine comme la protection de l'environnement, où le problème des externalités transfrontières occupe une place centrale, il a été démontré que la compétition législative internationale est plutôt de nature à entraîner sa dégénérescence vers le bas, chaque législateur étant tenté d'externaliser le coût de ses propres activités sous la pression du marché, dans l'effort d'attirer des investisseurs. Au mieux, il faudrait donc conclure que les bienfaits de la compétition législative sont à mesurer au cas par cas et dépendraient de l'étendue du problème des coûts sociaux dans chaque domaine spécifique. C'est ainsi que les partisans de la compétition de dérégulation en matière économique tirent argument de l'absence d'externalités dans des domaines tels que la régulation boursière.

Mais c'est là, qu'interfère la question de la communauté juridique, chaque législateur pouvant avoir sa propre vision de la finalité à poursuivre. Ainsi, par exemple, les analyses contemporaines du droit fédéral des « securities » attribuent à ce dernier une fonction protectrice des seuls intérêts privés des parties, la protection du marché lui-même, c'est-à-dire des intérêts des investisseurs en général, est refoulée à l'arrière-plan. Une démonstration analogue relative aux finalités variables des diverses régulations économiques peut être faite dans le champ du droit de la concurrence, où, par exemple, les législations de pays en voie de développement peuvent poursuivre des politiques économiques très 
différentes de celles qui inspirent les lois des pays développés. La fongibilité, condition de la libre concurrence législative, fait à nouveau défaut, et tout projet fondé sur la reconnaissance mutuelle s'avère dès lors illusoire. En effet, il n'est pas plus réaliste de croire que des Etats poursuivant des politiques économiques très différentes s'accorderont un jour spontanément sur les finalités de leurs législations qu'il ne l'est de penser qu'ils évolueront naturellement vers une acceptation partagée des " effets » que peut produire une activité exercée à l'étranger sur leur propre économie. Dans les deux cas, tant l'identification que l'attribution de la charge des externalités demeurent très problématiques.

L'affaire Lloyd's illustre en effet un phénomène intéressant, qui est celui de la dilution de l'impérativité des réglementations nationales de police - devenues selon le terme heureux d'un auteur, d'application seulement « semi nécessaire ». Ce phénomène résulte de l'admission de clauses de choix de for dans des domaines assujettis à des réglementations de police ; à cet égard, l'arbitrage du contentieux relevant de réglementations économiques a été l'impulsion décisive de cette évolution. Or, dès lors que la méconnaissance de la réglementation de police du for n'entrave pas la circulation de la décision, arbitrale ou étrangère, rendue sur le fondement de la clause, on relève une réduction significative de l'impact effectif de certaines règles de droit économique pourtant jugées « indérogeables » lorsqu'elles sont mises en œuvre directement par les autorités du for. Les pressions venues du marché induisent ainsi une forme spontanée d'autorégulation - en somme, une allocation efficiente des compétences législatives à la faveur du jeu des règles de conflits de juridictions.

Il est temps en effet de prendre acte des bouleversements épistémologiques qu'entraîne le nouvel enchevêtrement des économies, et d'admettre que le conflit de lois peut avoir un enjeu politique, tout comme les régulations nationales de droit public économique peuvent subir l'impact des volontés privées. Les mêmes soucis peuvent être transposés au marché du carbone et aux futurs contrats internationaux dont l'objet est la vente de ces crédits.

Repenser l'ordre juridique international en raison des phénomènes de mondialisation est un problème qui ne se pose bien, semble-t-il, que si on tient compte des deux notions fondamentales de l'histoire de la pensée qui sont au cœur des débats les plus contemporains. Il s'agit des notions d'Etat souverain et de communauté humaine mondiale. "Que l'Etat soit pensé comme une communauté humaine est en effet une idée acquise au moment de la formation du concept d'Etat lui-même. Prises ensemble, dans tous les cas, les notions de communauté humaine mondiale et étatique souverain semblent apparemment s'exclure. La souveraineté de l'un paraît constituer une barrière 
insurmontable à la réalisation véritable de l'autre ou, inversement, l'avènement de la seconde semble signer le déclin du premier ${ }^{26} »$.

La mondialisation comprend au niveau juridique un dédoublement des problèmes qui a été parfaitement exposé par CHEVALLIER ${ }^{27}$ : « il s'agit, en premier lieu, d'une mondialisation générale des phénomènes de mondialisation et donc du droit. Le droit est en effet lui-même englobé dans les phénomènes de mondialisation et donc peut-être en voie d'être mondialisé dans certains de ses domaines (paix, environnement, santé, démographie, etc..). Mais, en second lieu, la mondialisation des phénomènes sociaux, autres que le droit, appelle également le développement d'un droit qui lui soit propre et qui permet de la réguler en partie; d'où cette fois-ci l'idée d'un droit de la mondialisation $"$.

\section{Conclusion}

La gouvernance et les principes environnementaux sont des notions qui comportent plusieurs aspects. Le principe de responsabilité est le principe environnemental qui chapeaute l'application des notions de gouvernance dans un contexte de développement durable. La gouvernance idéale implique un médiateur entre l'institution publique et la société civile. L'association entre le principe de prévention et la gestion de crise met en œuvre la méthode délibérative avec la participation de la société civile ce qui permet une meilleure gouvernance. Toutefois, le principe de prévention a ses limites dans la gestion de crise car il s'avère qu'aucune réponse définitive et fiable n'est possible. Le principe de précaution vient alors compléter ces enjeux de gouvernance. Ce principe a fait couler beaucoup d'encre sur sa définition et son application. Néanmoins, il apparaît comme essentiel. Sa mise en œuvre effective n'est possible que sous certaines conditions de fond et de forme précises. Le principe de responsabilité appliqué aux différents contextes de gouvernance permet de se pencher sur les notions de provisoire et d'urgence. La notion de provisoire est pertinente dans le cas du juge des référés qui ne peut ordonner des mesures irréversibles qu'avec une extrême circonspection et que dans la mesure où il existe une évidence suffisante de bon droit. La notion d'urgence s'applique différemment selon le contexte et les définitions conditionnant son application dans les différentes juridictions mais il existe une urgence existentielle pour « la communauté internationale d'énoncer

\footnotetext{
${ }^{26}$ MUIR WATT, Horatia. Globalisation des marchés et économie politique du droit international privé. La mondialisation entre illusion et utopie. Archives de philosophie du droit - Tome 47 - Ouvrage publié avec le concours du CNRS. Dalloz, 2003. p. 191.

${ }^{27}$ CHEVALLIER, J. Mondialisation du droit ou droit de la mondialisation ?, Le droit saisi par la mondialisation, p.37 et suivante. Bruylant, pp. 37-61, 2001.
} 
des principes universels sur la base desquels l'humanité pourra répondre aux dilemmes et controverses de plus en plus nombreux que la science et la technologie suscitent pour l'humanité et l'environnement ${ }^{28}$ ". Enfin, nous ne pouvons pas laisser de côté le contexte économique qui nous soumet à certaines responsabilités à différents niveaux. Les bouleversements épistémologiques qu'entraîne le nouvel enchevêtrement des économies, conduisent à admettre que le conflit de lois peut avoir un enjeu politique, tout comme les régulations nationales de droit public économique peuvent subir l'impact des volontés privées. Les rapports entre les secteurs publics et les secteurs privés doivent être repensés dans le contexte de la mondialisation.

\section{Bibliographie}

\section{Conventions et textes de Loi}

Convention de Vienne du 22 mars 1985, puis Protocole de Montréal en septembre 1987.

Convention du Conseil de l'Europe sur « la responsabilité civile des dommages résultant d'activités dangereuses pour l'environnement ", 21 juin 1993.

Loi n 95-101 du 2 février 1995, relative au renforcement de la protection de l'environnement - Loi Barnier.

Loi n²000-597 du 30 juin 2000 relative au référé devant les juridictions administratives.

Unesco, Déclaration universelle sur la bioéthique et les droits de l’homme, 19 octobre 2005.

\section{Doctrine}

BAIL, Christophe. Environmental Governance : Reducing risks in democratic societies. Introduction paper, EEC, Future Studies Unit, 1996.

CHEVALLIER, J. Mondialisation du droit ou droit de la mondialisation ?, Le droit saisi par la mondialisation, Bruylant, pp. 37-61, 2001.

\footnotetext{
${ }^{28}$ Op. cit. $4^{e}$ consid.
} 
GODARD, Olivier. L'ambivalence de la précaution et la transformation des rapports entre science et décision. Éd. de la Maison des Sciences de l'Homme et INRA-Éditions, pp.37-83, 1997.

HERMITTE, Marie-Angèle cité par LARRERE, Catherine et LARRERE, Raphaël. Du bon usage de la nature. Pour une philosophie de l'environnement. Alto Aubier, Paris, 1997 (mars).

MEUR-FEREC, Catherine. La GIZC à l'épreuve du terrain : premier enseignements d'une expérience française., en ligne : <www.developpementdurable.revues.org/index4471.html>

MUIR WATT, Horatia. Globalisation des marchés et économie politique du droit international privé. La mondialisation entre illusion et utopie. Archives de philosophie du droit - Tome 47 - Ouvrage publié avec le concours du CNRS. Dalloz, 2003.

RIST, Gilbert. Le développement. Histoire d'une croyance occidentale. Presses de Sciences Po, Paris, 1996.

SUNDERLIN, W. D. Global environmental change, sociology and paradigm isolation. Global environmental change, vol. 5, number 3, Juin 1995.

\section{Documents}

Rapport Brundtland, Notre avenir à tous, Montréal, éd. du Fleuve, 1988.

OMC, Rapport de l'Organe d'appel, Corée - Mesures affectant les importations de viande de bœuf fraîche, réfrigérée et congelée, WT/DS161/AB/R et WT/DS169/AB/R, adopté le 10 janvier 2001.

Trabalho enviado em $x x$

Aceito em 04 de fevereiro de 2019 